\title{
Apparatus for Dynamical Texture Measurements by Neutron Diffraction Using a Position Sensitive-Detector
}

\author{
D. JUUL JENSEN and J. K. KJEMS \\ Ris申 National Laboratory, Roskilde, Denmark
}

(Received August 27, 1982; in final form November 22, 1982)

\begin{abstract}
A new apparatus for dynamical texture measurements using neutron diffraction has been developed. A variable wavelength neutron spectrometer has been modified to incorporate a linear position-sensitive detector. The sample is orientated by a fully automatic Euler goniometer, and it can be heated by a stream of hot air at rates up to $200^{\circ} \mathrm{C} / \mathrm{min}$. A quarter of a complete pole figure can be recorded in 14 minutes with an accuracy, determined by counting statistics, of 2-3\%. The experimental set-up is described in the present paper and the performance, including sources of systematical errors, is discussed. Finally the viability of this apparatus is demonstrated by studies of the recrystallization kinetics of $95 \%$ cold rolled copper.
\end{abstract}

\section{INTRODUCTION}

The determination of texture by diffraction is an important part of the characterization of metals and alloys (Barrett and Massalski, 1966); both $\mathrm{x}$-ray and neutron-diffraction are commonly used for this purpose. Up to now these methods have mainly been applied to static studies, and only recently have they been extended to kinetic studies. The potential of neutron diffraction as a tool for the accurate investigation of recrystallization kinetics of individual texture-components has been demonstrated by Hansen et al. (1981). Based on this work, a new apparatus for fast pole figure measurements has been developed modifying a neutron spectrometer by an Euler goniometer and a linear position-sensitive detector. The potential of the apparatus has been used to study the recrystallization kinetics of fine and coarse-grained heavily rolled pure copper (Juul Jensen et al., 1982). In this case a quarter of a complete pole figure, based on a sampling mesh of $61 * 19$ measuring 
points, can be recorded in 14 minutes with an accuracy of $2-3 \%$ as determined by counting statistics. The sample size was $1 / 2 \mathrm{~cm}^{3}$.

\section{EXPERIMENTAL SET-UP}

\section{The spectrometer}

The experiments were carried out with neutrons from the Ris $\phi$ DR3 reactor. A TAS7 spectrometer, placed in an experimental hall outside the reactor containment, was used; the neutron beam is transferred to the hall via a $20 \mathrm{~m}$ long neutron guide. A variable wavelength can be selected by a curved pyrolytic graphite crystal monochromator, which produces a vertically focused beam on the sample. The flux at the sample position is approximately $5 \times 10^{6} \mathrm{n} / \mathrm{cm}^{2} / \mathrm{sec}$ at a neutron wavelength of $3.0 \AA$. . The sample is mounted in an Euler goniometer, where it can be rotated about three axes under computer control with an accuracy of 1/100 degree (Figure 1). The diffracted beam is detected by a linear position-sensitive detector, placed with its axis vertical. This allows the determination of the scattered intensity along a Debye-Scherrer ring, and hence it leads to a considerable enhancement of the data collection rate. With a scattering angle $2 \theta_{h k l}$, between the incident and the diffracted beam, equal to 90 degree the Debye-Scherrer cone becomes a plane perpendicular to the incident beam (Figure 2), meaning that one and the same diffraction condition is fulfilled along the entire detector. This implies that with one setting of the sample, the detector simultaneously scans several degrees of a small circle in an $(\mathrm{hkl})$-pole figure. In the present set-up the detector covers 34 degrees, when it is projected onto the pole figure plane. For most low index reflections from common metals and alloys the continuous spectrum from a thermal reactor allows one to choose a wavelength such that $2 \theta_{h k l}=90^{\circ}$.

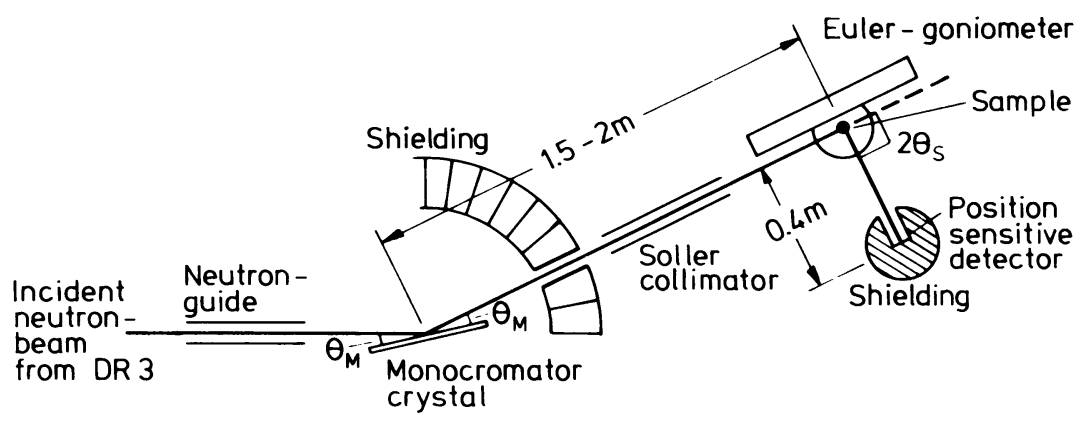

FIGURE 1 Schematic layout in the horizontal plane. 


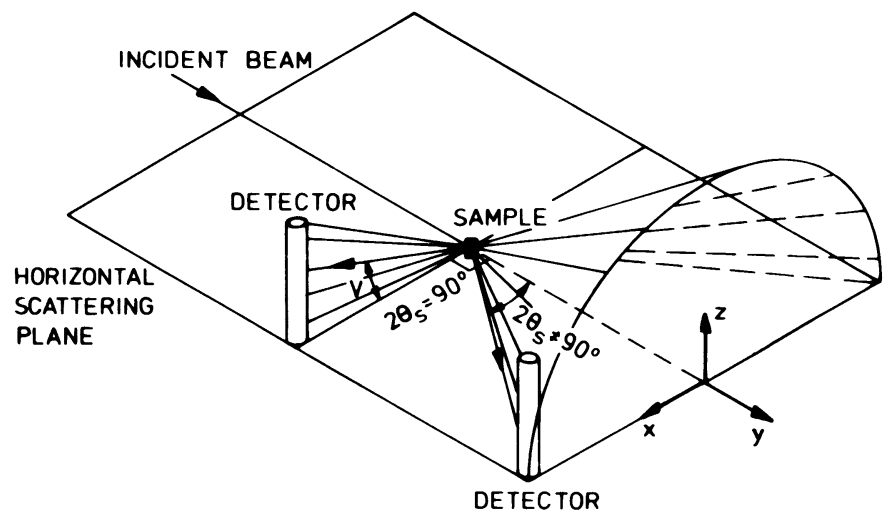

FIGURE 2 Illustration of the use of a position-sensitive detector to measure the intensity distribution along a $(h k l)$-Debye-Scherrer cone. At $2 \theta_{h k l}=90^{\circ}$ the cone degenerates to a plane, and the detector axis lies completely in this plane.

\section{The detector}

A proportional gas detector can be made position-sensitive, when it is equipped with a resistant anode wire (Kjems, 1970). The present detector is a $45 \mathrm{~cm}$ long, $5 \mathrm{~cm}$ diameter stainless steel tube with a filling of $2 \mathrm{~atm} \mathrm{He}+$ $0.8 \mathrm{~atm} \mathrm{Kr}(\mathrm{STP})$. The resistant anode $(2.5 \mathrm{k} \Omega / \mathrm{cm})$ is a quartz wire, $0.3 \mathrm{~mm}$ diameter and coated with colloidal graphite. The neutrons are detected via the ${ }_{0}^{1} \mathrm{n}+{ }_{2}^{3} \mathrm{He} \rightarrow{ }_{1}^{1} \mathrm{P}+{ }_{1}^{3} \mathrm{~T}+764 \mathrm{keV}$ nuclear reaction which leads to the standard proportional gas counter response. The position of the neutron impact in the detector is determined by the difference in rise time for pulses arriving at each end of the anode wire. The anode resistance together with the detector capacitance gives the characteristic time constant. The actual timing is based on the times when the doubly differentiated signals cross through zero from the charge sensitive preamplifiers at each end of the detector (Figure 3). A delay amplifier is used to ensure that the signals from one end always arrive first to the time analyzer (TAC). Ideally, there is a linear relation between the impact position and the observed time difference:

$$
X \simeq\left(t_{2}-t_{1}+t_{D}\right) /\left(R_{T} C_{T}\right)
$$

where $X$ is the position, $t_{1}$ and $t_{2}$ are the zero-crossing times for the differentiated signals from each end of the detector, $t_{D}$ is the delay time, $R_{T}$ the total anode resistance and $C_{T}$ the total capacitance. The pulses from the TAC are analysed in the multi channel analyser (MCA), which uses 128 channels. The position resolution of the detector system is about $1 \mathrm{~cm}$ full width half maximum, and it can handle counting rates up to $20000 \mathrm{cps}$ without appreci- 


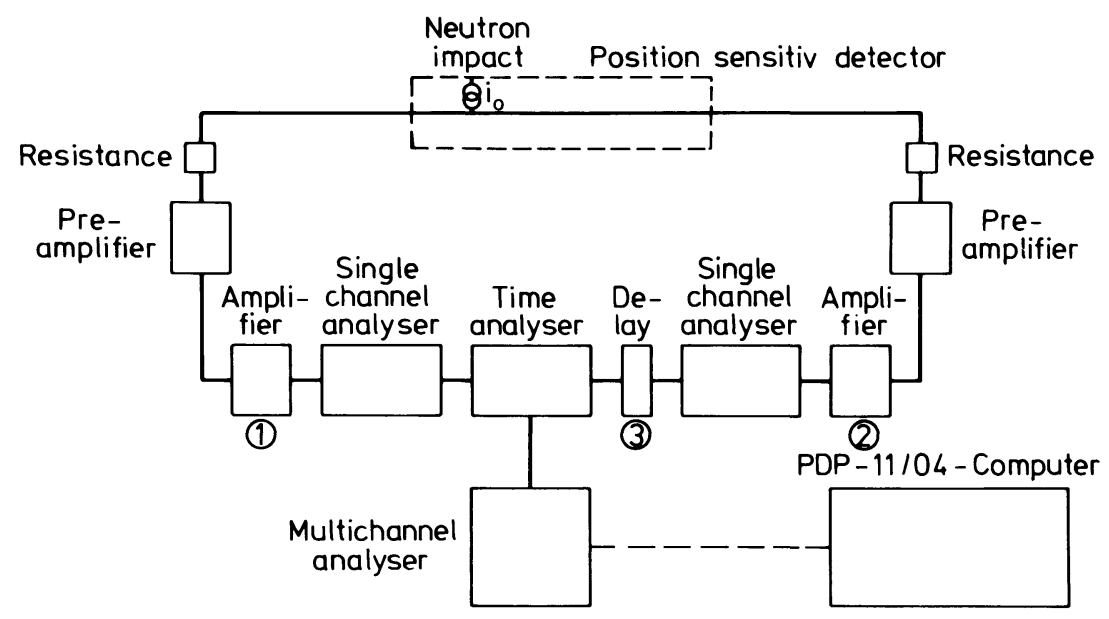

FIGURE 3 Layout of the electronics for the linear position-sensitive detector.

able dead time (if a higher counting rate is wanted, it is possible to correct for the deadtime via the MCA system).

The calibration of the detector involves both the sensitivity and the linearity i.e. the detector efficiency and the relation between a channel number in the MCA and the corresponding $X$. The calibration is carried out by placing an incoherently scattering vanadium sample at the sample position and recording the response with different neutron-absorbing cadmium masks in front of the detector. This gives a simultaneous determination of the linearity and the sensitivity. For the present use, the 128 MCA-channels are summed to give 19 equivalent segments or reduced channels along the detector. This means that one can regard the position-sensitive detection system as 19 independent detectors (reduced detector channels) each covering a 2.67 degree section of the Debye-Scherrer circle and a solid angle of $8 \times 10^{-4}$ steradians in the present set-up, where the detector is placed $38 \mathrm{~cm}$ from the sample.

\section{Samples and the furnace}

The advantage of neutron diffraction measurements for texture determination is the freedom to use many sample geometries, since the neutron beam penetrates even bulk samples. For the first experiments we used copper samples with a nominal purity of $99.99 \%$ and with two different grain sizes $35 \mu \mathrm{m}$ and $0.5-1 \mathrm{~mm}$. Both materials were cold rolled to $95 \%$ reduction. Circular discs with a diameter of $1.5 \mathrm{~cm}$ were stamped out and chemically polished to suppress the possible contribution from the surface texture. Six 
discs were tied together with a Mo-wire resulting in a cylindrical sample with a height of approximately $3 \mathrm{~mm}$.

The sample temperature is measured with a chromel-alumel thermocouple placed in the centre of the sample. The heating of the sample is performed by a stream of hot air, and the current through the heating elements in the air-blower is controlled by a thor-regulator connected to the thermocouple. With a separation of $2 \mathrm{~cm}$ between the furnace and the sample a typical heating time to $200^{\circ} \mathrm{C}$ is 1 minute after which the temperature is stable to within $1^{\circ} \mathrm{C}$ as measured in the sample centre. Because of the fast heating rate this furnace is very suitable for investigations of recrystallization kinetics in the low temperature regime. For temperatures below $300^{\circ} \mathrm{C}$ the temperature gradient through the copper-sample is less than $2^{\circ} \mathrm{C}$, rising to $10^{\circ} \mathrm{C}$ at $400^{\circ} \mathrm{C}$.

\section{Computer control}

The complete spectrometer, the temperature controller and the processing of the signals from the position-sensitive detector system are controlled by a PDP-11/04 computer. The programs for the pole figure measurements are written in BASIC, and the procedure is fully automatic. The measured data are stored on standard floppy discs, and they can be analysed on any PDP-11 computer or transferred to a more powerful computer. Besides controlling the measurements, the PDP-11 computer conducts an automatic plotting of the resultant pole figures on a 4 colour HP-plotter. Each colour indicates a pole density level given in preselected units of random density.

\section{MEASURING PROCEDURE}

A set of measured intensities along the detector is shown in Figure 4a. For a given setting of the sample, this corresponds to a part of a small circle in the pole figure as shown in Figure $4 \mathrm{~b}$ where each dot represents a reduced detector channel. The calculation of the correspondence is based on the definition of the Euler-angles and is shown in the appendix. By rotating the sample the complete pole figure or part of it can be covered. In Figure $4 \mathrm{c}$ this is shown for a quarter of a pole figure, where each "ring" is obtained by rotating the sample about a direction normal to it. The different "rings" corresponds to different $\phi_{1}, \Phi$ settings.

TABLE I. Actual angle settings in degrees.

\begin{tabular}{rrr}
\hline$\phi_{1}$ & \multicolumn{1}{c}{$\Phi$} & $\Delta \phi_{2}$ \\
\hline 45 & 90 & 10 \\
0 & 28 & 5 \\
0 & -15 & 5 \\
\hline
\end{tabular}




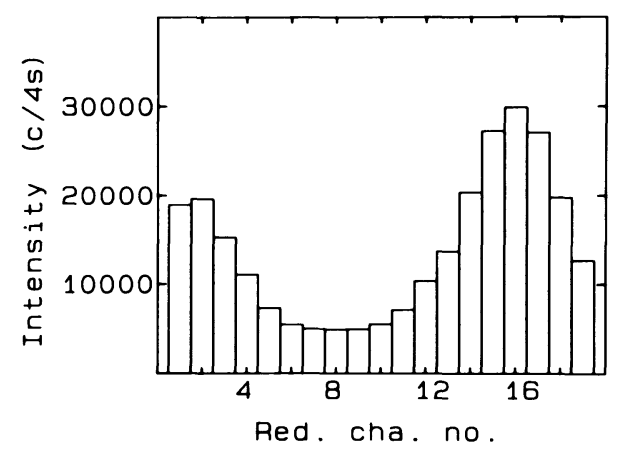

(a)

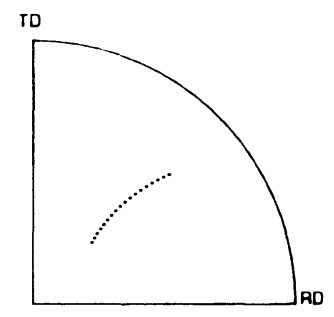

(b)

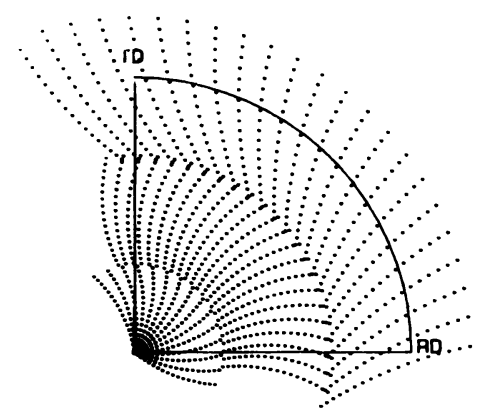

(c)

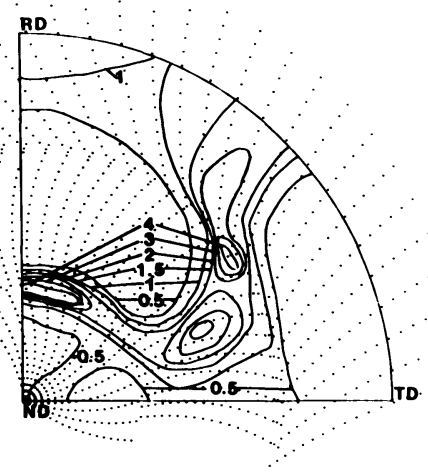

(d)

FIGURE 4 a) Measured intensities along the detector.

b) With one sample setting $51^{\circ}$ along a small circle are covered by the detector.

c) The sampling mesh.

d) A measured (200)-pole figure for coarse-grained copper rolled $95 \%$.

As may be seen in Figure 4c there is some overlap between the "rings", in order to ensure that the pole density measured at one end of the detector corresponds to that measured for the same point at the other end of the detector with another setting of the sample. Furthermore, the points near the edge of the pole figure are measured twice, or, as it is plotted, some points are measured outside the edge of the figure; this allows a check that the pole figure has the required symmetry. 
An example of a measured pole figure is shown in Figure 4d, where the contour lines are used to distinguish the different colors.

\section{SOURCES OF SYSTEMATIC ERRORS}

To quantify the meaning of the contour levels in the pole figure, the measured intensities has to be normalized and corrected for background. The normalization is carried out either by integrating over the complete pole figure or by comparing the actual pole figure with a random sample pole figure measured under exactly the same experimental conditions. In the first case the measured intensities have to be corrected for absorption, extinction and in the high temperature region also for the Debye-Waller factor (Bacon, 1975).

\section{Intensity correction}

The absorption calculation $I=I_{0} A$ is based on the formula

$$
A=\frac{1}{V} \int \exp \left(-\mu\left(1_{i}+1_{r}\right)\right) \mathrm{d} V
$$

where

$\mu \quad$ is the linear absorption coefficient

$1_{i}+1_{r}$ is the total length of the neutron path inside the sample

$V \quad$ is the volume of the sample.

The calculation is done numerically, based on the assumptions that the incident beam is well collimated and the sample is small seen from a reduced detector channel. The values $1_{i}, 1_{r}$ are found by transferring the directions of the incident and the reflected beam to the sample coordinate system and finding the intersection with the sample cylinder surface. This absorption calculation has to be carried out for every angle $V$ to a reduced channel along the detector and for all $\phi_{1}, \Phi$ settings of the sample.

Corrections for primary extinction are not needed if $m q \ll 1$, where $m$ is the number of planes in one mosaic block, and $q$ is the amplitude reflected by a single plane (Bacon, 1975).

$$
q_{h k l}=N_{c} d \frac{\lambda}{\sin \theta_{h k l}} F_{h k l}
$$

where $N_{c}$ is the number of unit cells per unit volume, $d$ is the interplanar spacing of the $(h k l)$-planes and $F_{h k l}$ is the structure factor.

For the (200)-reflection in copper the size of a perfect mosaic block has 
to be much less than $4.2 \mu \mathrm{m}$, if primary extinction is to be negligible. This figure refers to perfect crystallites which are dislocation free, and in heavily rolled materials, where the dislocation density is high (the subgrain size is smaller than $1 \mu \mathrm{m}$ ), one would not expect to find primary extinction.

The correction for secondary extinction is effected by replacing the value for the absorption cross section in the calculations of the linear absorption coefficient by the total cross section (values can be found in tables e.g. ref $6)$.

The Debye-Waller correction $I=I_{0} e^{-2 w}$, where the exponential term is due to thermal vibration, is negligible for the present copper-experiments, but could be important for other materials and/or higher temperatures.

\section{Background}

The background is measured by scanning the detector through the Bragg peak, thus changing the scattering angle. The approximately linear background has 3 contributions: thermal diffuse scattering(TDS), multiple scattering and incoherent scattering. For a single crystal the TDS peaks at the same angular position as the Bragg-peak, but for a polycrystal, where many crystals, not in the Bragg position, contribute to the TDS, this peak tends to smear out. The size of the TDS could be estimated by measurements at liquid nitrogen temperature. Estimates for the background due to incoherent and multiple scattering may be calculated from the incoherent and the total scattering cross sections respectively.

\section{The detector resolution}

The instrumental resolution function can be experimentally determined by the Bragg diffraction from a single crystal specimen at the sample position. The resolution for each detector element is defined as the half value intensity contour as a function of the misalignment in Euler angles for Bragg scattering in the detector element in question. This is measured by keeping $\phi_{1}$ fixed at $90^{\circ}$ and scanning $\Phi, \phi_{2}$ giving the contribution along and perpendicular to the detector, respectively. The measured resolution ellipse for 3 different reduced channels is shown in Figure 5a. Due to the vertical divergence of the beam, the size and the tilt between the resolution ellipse and the direction along the detector increases for diffraction out of the horizontal plane. The appropriate diameters of the ellipse observed in reduced channel 6 are approximately $6^{\circ}$ along and $2^{\circ}$ perpendicular to the detector. The measured resolution function refers to the experimental set-up, and is independent of the sample orientation. By way of illustration, the projection of typical resolution ellipses on the pole figure plane are shown in Figure $5 \mathrm{~b}$. 


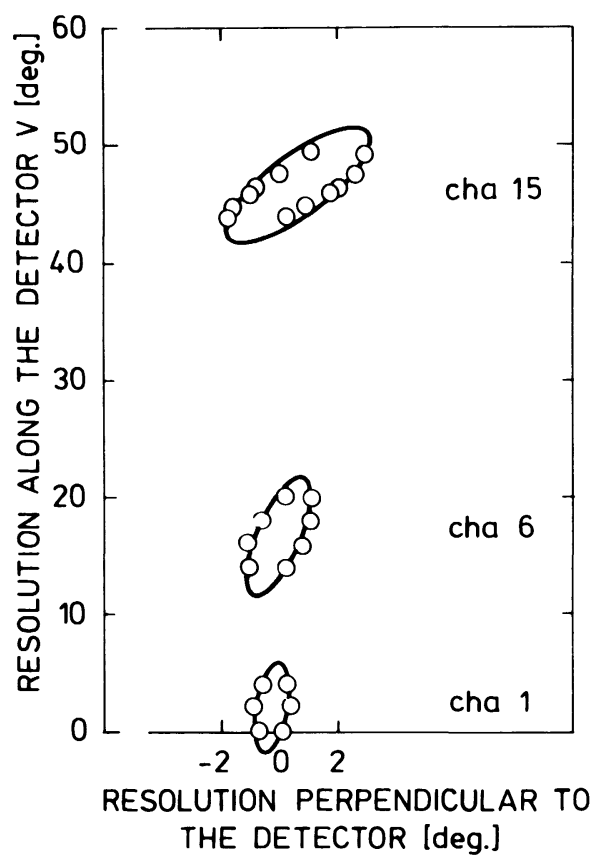

(a)

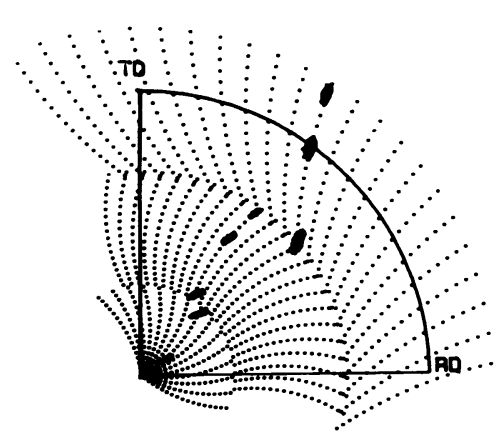

(b)

FIGURE 5 a) The resolution function for selected detector elements.

b) Projection of typical resolution ellipses to the pole figure plane.

\section{DYNAMIC MEASUREMENTS}

\section{Recording time}

At the present stage a quarter of a complete pole figure can be recorded in 14 minutes, with an accuracy, determined by counting statistics, of $2-3 \%$. Of these 14 minutes only 3 are spent counting, while the rest are used for setting the sample orientation. With more powerful motors on the Eulergoniometer, the setting time would be substantially reduced, and we would expect to get to a measuring time of 8 minutes for the pole figure described. This time interval (14 or 8 minutes) places an apparent limit on the rate of any kinetic process which may be studied. However faster processes can be followed through partial pole figures which focus on selected texture components. Meaning the sample orientation is only changed between a few preselected positions. In the limiting case both sample and detector can be kept fixed during the measurements resulting in a simultaneous scan along 
$34^{\circ}$ of a small circle in the pole figure. In this manner events with time constants of the order of minutes can be followed.

\section{Recrystallization experiments}

The development of the (200) and the (111) pole figure for both fine- and coarse-grained copper were followed during isothermal anealing at several different temperatures. The temperature intervals from $123^{\circ} \mathrm{C}$ to $175^{\circ} \mathrm{C}$ and from $177^{\circ} \mathrm{C}$ to $195^{\circ} \mathrm{C}$ were used for the fine- and the coarse-grained material respectively. To achieve investigations with better time resolution than the 14 minutes used for complete pole figure measurements, experiments were also performed where only partial pole figures were recorded. An example of this is shown in Figure 6, here the evolution of two texture components in the (200) pole figure is given. Because of the position-sensitive detector it is possible to detect during the anealing both the decreasing maximum in the rolled texture near the ND-RD line (see Figure 4d) and the increasing peak at the center without changing the sample position. This example demonstrates the versatility and sensitivity of this instrument for studying recrystallization kinetics.

The data has been analysed by reference to the Avrami equation (Avrami, 1940) and the results have been discussed elsewhere (Juul Jensen, 1982).

\section{DISCUSSION}

A fast in-situ texture-measuring technique based on neutron diffraction has been developed. A variable wavelength neutron spectometer specially equipped with

1) an Euler goniometer, where the sample can be orientated as desired

2) a linear position-sensitive detector placed along a Debye-Scherrer ring

With this instrument it is possible to measure complete pole figures for most low index reflections from common metals and alloys. For a $1 / 2 \mathrm{~cm}^{3}$ copper-sample a quarter of a complete pole figure is measured in 14 minutes. If events with time constants shorter than 1 hour are of interest, their kinetics may be studied by measuring the evolution of specific texture components in the pole figure.

The measuring procedure and the plotting of the results are fully automatic, and conducted by a PDP-11 computer. The data are stored on standard floppy discs, and are easily transferred to more powerful computers. It is our aim to synthesize the orientation distribution function (ODF) (Bunge, 1969) based on the measured pole figures, and then be able to follow the evolution of the ODF during kinetic processes. 


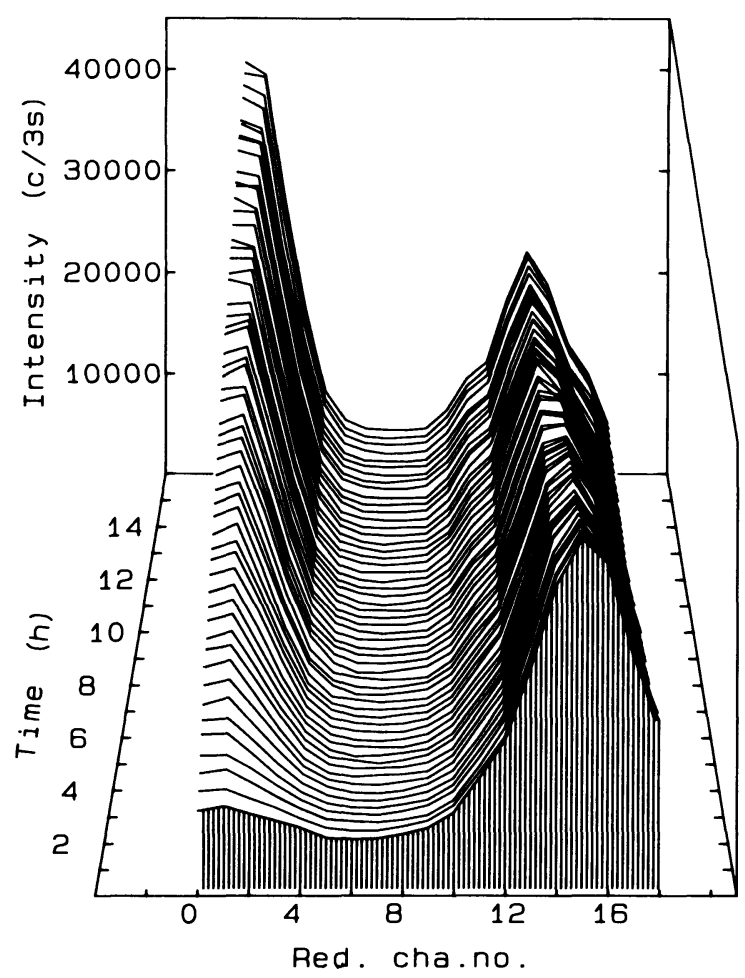

FIGURE 6 The evolution of 2 texture components in the (200)-pole figure for fine-grained pure copper, measured with the sample fixed in one position.

\section{References}

Avrami, M. J. Chem. Phys. 8, 212 (1940).

Bacon, G. E. Neutron Diffraction. 3rd ed. Clarendon Press, Oxford (1975).

Barrett, C. S. and Massalski, T. B. Structure of Metals. 3rd ed. McGraw-Hill, New York (1966).

Bunge, H. J. Matematische Methoden der Texturanalyse. Akademie-Verlag, Berlin (1969).

Hansen, N., Leffers, T. and Kjems, J. K. Acta met. 29, 1523 (1981).

Hughes, D. J. and Schwartz, R. B. Neutron cross sections. Brookhaven National Lab. New York (1958).

Juul Jensen, D., Hansen, N., Kjems, J. K. and Leffers, T. Proc. Sixth International Conference on Materials, 1179 (1982).

Kjems, J. K. Report 229, Risø National Lab. Denmark (1970). 


\section{Appendix}

\section{CORRESPONDANCE BETWEEN THE SAMPLE ORIENTATION AND THE POLE FIGURE POINT}

To establish the correspondance between the sample orientation and the pole figure two coordinate systems are used. The laboratory system is defined with the $x$-axis along the diffracted beam in the horizontal plane, the $y$-axis along the incident beam and the $\mathrm{z}$-axis in the vertical direction perpendicular to the $x$ - and $y$-axis. The sample system is defined with the $x^{\prime}$-axis along the rolling direction, the $y^{\prime}$-axis along the transvers direction and the $z^{\prime}$-axis along the normal direction. Both systems have the origin in the center of the sample.

Using Euler angles, the transformation of the laboratory system into the sample system, is given by three consecutive rotations (Figure $7 \mathrm{a}$ ). The rotation matrix has the form:

$$
\begin{aligned}
{\left[\begin{array}{l}
\mathrm{x}^{\prime} \\
\mathrm{y}^{\prime} \\
\mathrm{z}^{\prime}
\end{array}\right]=\left[\begin{array}{rrr}
\cos \phi_{2} & \sin \phi_{2} & 0 \\
-\sin \phi_{2} & \cos \phi_{2} & 0 \\
0 & 0 & 1
\end{array}\right] } & {\left[\begin{array}{rrr}
1 & 0 & 0 \\
0 & \cos \Phi & \sin \Phi \\
0 & -\sin \Phi & \cos \Phi
\end{array}\right] } \\
& {\left[\begin{array}{rrr}
\cos \phi_{1} & \sin \phi_{1} & 0 \\
-\sin \phi_{1} & \cos \phi_{1} & 0 \\
0 & 0 & 1
\end{array}\right]\left[\begin{array}{l}
\mathrm{x} \\
\mathrm{y} \\
\mathrm{z}
\end{array}\right] }
\end{aligned}
$$

or $\bar{r}^{\prime}=\bar{T}\left(\phi_{1}, \Phi, \phi_{2}\right) \bar{r}$

where the "primed system" $\bar{r}^{\prime}=\left(\mathrm{x}^{\prime}, \mathrm{y}^{\prime}, \mathrm{z}^{\prime}\right)$ corresponds to the sample-system.

The relation between the observed intensity in one channel of the positionsensitive detector and the point in the pole figure is found by the following procedure: The intersection between the normal to the $(h \mathrm{kl})$-scattering plane and a unit reference sphere circumscribed around the sample is at a pole $Q$. The corresponding pole in the pole figure is the stereographic projection of this pole $Q$ into the pole figure plane (Figure 7b). In the laboratory-system $Q$ is given by

$$
\bar{r}_{Q}=\left[\begin{array}{c}
\mathrm{x}_{Q} \\
\mathrm{y}_{Q} \\
\mathrm{z}_{Q}
\end{array}\right]=\left[\begin{array}{cc}
\sin 45^{\circ} & \cos V \\
-\cos 45^{\circ} \\
\sin 45^{\circ} & \sin V
\end{array}\right)
$$

where $V$ is the angle between the horizontal plane and the direction to the actual reduced detector channel (Figure 2). With the detector position chosen in these experiments the angle $V$ varies from $0^{\circ}$ to $51^{\circ} . Q$ is transformed to 


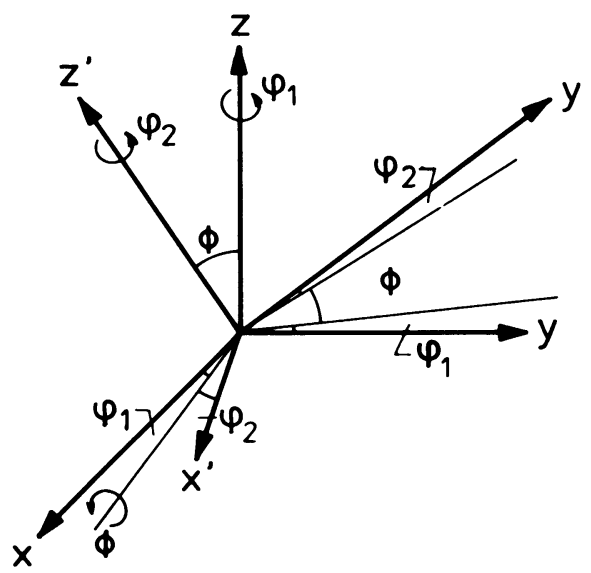

(a)

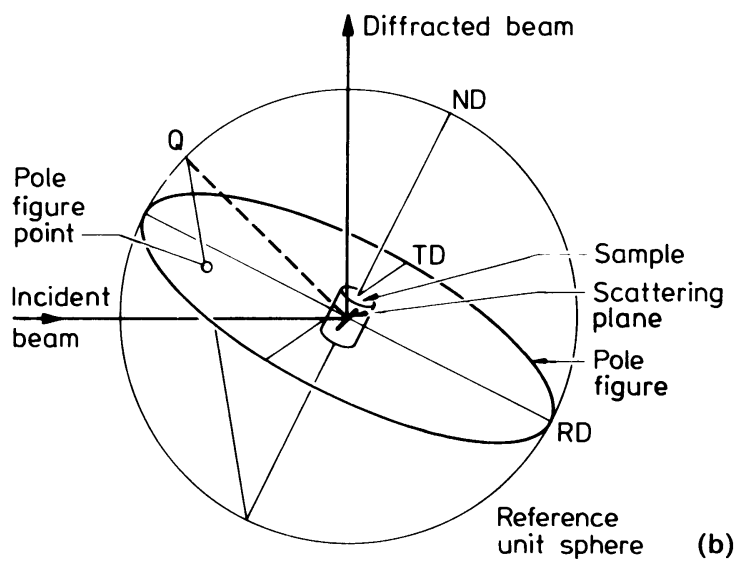

FIGURE 7 a) Definition of the Euler angles.

b) Definition of the pole figure.

the sample system $\bar{r}^{\prime}=\overline{\bar{T}}\left(\phi_{1}, \Phi, \phi_{2}\right) \bar{r}_{Q}$ and the pole figure point is found to be

$$
\mathrm{x}_{p}^{\prime}=\frac{ \pm \mathrm{x}_{Q}^{\prime}}{\mathrm{z}_{Q}^{\prime} \pm 1} \quad \mathrm{y}_{p}^{\prime}=\frac{ \pm \mathrm{y}_{Q}^{\prime}}{\mathrm{z}_{Q}^{\prime} \pm 1}
$$

